\title{
Expression of novel cancer/testis antigen TMEM31 increases during metastatic melanoma progression
}

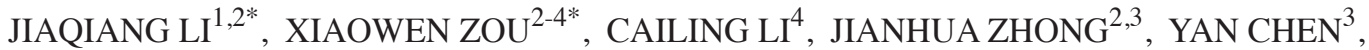

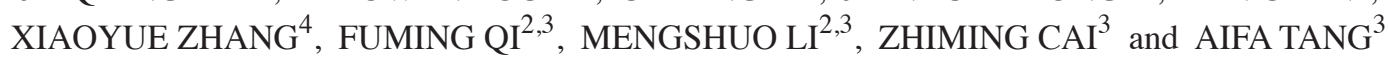 \\ ${ }^{1}$ Department of Pediatric Urology, Shenzhen Children's Hospital, Shenzhen, Guangdong 518026; \\ ${ }^{2}$ Shantou University Medical College, Shantou, Guangdong 515063; ${ }^{3}$ Department of Science and Education, \\ The Second People's Hospital of Shenzhen, The First Affiliated Hospital of Shenzhen University, Shenzhen, \\ Guangdong 518035; ${ }^{4}$ Guangdong and Shenzhen Key Laboratory of Male Reproductive Medicine and Genetics, \\ Institute of Urology, Peking University Shenzhen Hospital, Shenzhen, Guangdong 518036, P.R. China
}

Received October 8, 2015; Accepted November 30, 2016

DOI: $10.3892 / \mathrm{ol} .2017 .5728$

\begin{abstract}
Cancer/testis (CT) antigens are promising targets for immunotherapy due to their restricted expression in the germ cells of the testis in healthy tissue and high immunogenicity. The aim of the present study was to determine whether transmembrane protein 31 (TMEM31) is a CT antigen and to investigate the pattern of TMEM31 expression during the progression of melanoma. The pattern of expression of human TMEM31 mRNA in multiple human tissues was determined through reverse transcription-polymerase chain reaction analysis. TMEM31 protein expression was analyzed in the human testis, in addition to 128 primary melanoma and 64 metastatic melanoma samples through immunohistochemistry analysis. TMEM31 was identified to be predominantly expressed in the testis and weakly expressed in the placenta. In addition, TMEM31 protein expression was detected in 120/190 (63.16\%) melanoma samples (primary and metastatic). The intensity of TMEM31 staining in metastatic and primary melanomas was determined through semiquantitative integrated optical density (IOD) analysis, and identified to be significantly increased in metastatic melanoma compared with primary melanoma $(0.24 \pm 0.03$ vs. $0.09 \pm 0.01 \mathrm{IOD} / \mathrm{area} ; \mathrm{P}<0.001)$. The expression of TMEM31 protein was significantly increased in metastatic compared with primary melanoma samples $(76.56$ vs. $56.35 \% ; \mathrm{P}=0.017)$. The results of the present study suggest
\end{abstract}

Correspondence to: Dr Aifa Tang, Department of Science and Education, The Second People's Hospital of Shenzhen, The First Affiliated Hospital of Shenzhen University, 3002 Sungang West Road, Shenzhen, Guangdong 518035, P.R. China

E-mail: tangaifa2004@163.com

${ }^{*}$ Contributed equally

Key words: melanoma, cancer/testis antigens, transmembrane protein 31 , metastases, immunotherapy that TMEM31 is a novel CT antigen that serves an essential role in melanoma metastasis, in addition to being a potential immunotherapeutic target for the treatment of patients with melanoma.

\section{Introduction}

Melanoma is the most frequently occurring skin malignancy and the most common cause of skin cancer-associated mortality (1). Metastases are the most important prognostic factor for patients with melanoma (2). Studies identifying factors that predict the metastatic potential of melanomas have previously been performed (2). However, although numerous efforts have focused on understanding the processes of malignant transformation, progression and metastasis in melanoma, attempts to control melanoma metastasis have been unsuccessful (3). Thus, there is a need for the development of novel therapies for the treatment of advanced metastatic melanoma. The identification of novel diagnostic factors and therapeutic targets is required to aid in the diagnosis and treatment of patients with metastatic melanoma.

Cancer/testis (CT) antigens are expressed in a variety of different tumor types, but are exclusively expressed in testis germ cells and the placenta in healthy tissue (4-6). Due to their restricted expression pattern in healthy tissue, aberrant expression in various types of cancer and strong immunogenicity, CT antigens have been used in several cancer biomarker identification studies and therapeutic vaccination clinical trials $(7,8)$. The first human cancer antigen recognized by cytolytic $\mathrm{T}$ lymphocytes was a $\mathrm{CT}$ antigen that originated from a patient with melanoma in 1991 (8). As of 2008, >100 CT antigen genes have been identified, including CT antigen 1B (NY-ESO-1), sarcoma antigen 1, melanoma antigen (MAGE) A, the G antigen and SSX family members $(9,10)$. The majority of previous studies on CT antigens focused on assessing the expression patterns of these antigens in melanoma cells (11-14). However, little is known about the association between $\mathrm{CT}$ antigen expression and the clinical characteristics that distinguish primary tumors and metastases (14). This area warrants investigation, in order to 
identify potential biomarkers and immunotherapeutic targets to improve the efficacy of melanoma management.

Previously, the diverse expression patterns of human genes between the testes and other organs were investigated using the UniGene database (National Center for Biotechnology Information, Bethesda, MD, USA; www.ncbi.nlm.nih. gov/unigene) and its digital differential display tool (13). This led to the identification of 292 human testis-specific genes. Transmembrane protein 31 (TMEM31) was one of the human testis-specific genes identified and is located on chromosome $X$ (q22.2). In the present study, the expression profiles of TMEM31 in healthy human tissues and a series of different malignant tumor tissues were compared to investigate if TMEM31 is a novel CT antigen. Furthermore, an investigation into the expression characteristics of TMEM31 protein in 128 primary melanomas and 64 metastatic melanomas was performed, which will aid in understanding the progression of metastatic melanoma.

\section{Materials and methods}

Reverse transcription-polymerase chain reaction (RT-PCR). Total RNA was obtained from Takara Biotechnology Co., Ltd. (Dalian, China) for the following healthy tissues: Testis, placenta, lung, spleen, heart, skeletal muscle, prostate, stomach, small intestine, uterus, colon, bone marrow, brain, kidney, liver, thyroid, ovary and bladder. A total of $2 \mu \mathrm{g}$ of total RNA was reverse transcribed into single-stranded cDNA in $20 \mu \mathrm{l}$ of reaction buffer using Moloney-murine leukemia virus (M-MLV) reverse transcriptase (Thermo Fisher Scientific, Inc., Waltham, MA, USA). Forward and reverse oligonucleotide primers specific to TMEM31, and GAPDH were designed using Primer Express version 3.0 software (Applied Biosystems; Thermo Fisher Scientific, Inc.) and were synthesized by Sangon Biotech Co., Ltd. (Shanghai, China). The cDNA was amplified via PCR using the following primers: TMEM31 forward, 5'-GCCCAACAACTCTAATGCACC-3'; TMEM31 reverse, 5'-AGGTTGATCGTTCTGTCGCTG-3'; GAPDH forward, 5'-AGAAGGCTGGGGCTCATTTG-3'; GAPDH reverse, 5'-AGGGGCCATCCACAGTCTTC-3'. PCR reactions were performed in $20 \mu \mathrm{l}$ reaction mixtures containing PCR buffer, $2 \mathrm{mM} \mathrm{MgCl}_{2}, 0.4 \mathrm{mM}$ of each dNTP (dATP, dTTP, dCTP, dGTP), 0.5 units of the DNA polymerase (BioTools, Madrid, Spain), $0.4 \mu \mathrm{M}$ of each primer and $1 \mu \mathrm{l}$ of cDNA. PCR thermocycling conditions consisted of a precycling step at $95^{\circ} \mathrm{C}$ for $5 \mathrm{~min}$, followed by 35 cycles of denaturation at $95^{\circ} \mathrm{C}$ for $30 \mathrm{sec}$, annealing at $58^{\circ} \mathrm{C}$ for $30 \mathrm{sec}$ and extension at $72^{\circ} \mathrm{C}$ for $45 \mathrm{sec}$, with a final extension at $72^{\circ} \mathrm{C}$ for $10 \mathrm{~min}$. The PCR products were analyzed using agarose gel $(1.5 \%)$ electrophoresis and monitored under ultraviolet light. GADPH was used as a loading control.

Immunohistochemical staining. A paraffin-embedded malignant melanoma tissue microarray (\#ME2082), including details of patient gender, age, organ, pathology diagnosis, stage, tumor-node-metastasis stage and type, as well as a human testis tissue microarray (\#TEN241), including details of patient gender and age, were purchased from US Biomax Inc. (Xi'an, China) and analyzed using immunohistochemistry. Two primary melanoma samples were eliminated due to core tissue damage. TMAs were deparaffinized in xylene and rehydrated through treatment with a descending series of ethanol (100, 95 and $70 \%(\mathrm{v} / \mathrm{v})$ in double-distilled $\mathrm{H}_{2} \mathrm{O}$ ) and PBS washes. The sections were subsequently subjected to thermal antigen retrieval by boiling in citrate buffer $(2 \mathrm{mM}$ citric acid, $10 \mathrm{mM}$ sodium citrate, $\mathrm{pH}$ 6.0) for $15 \mathrm{~min}$. Following washes with PBS, endogenous peroxidases were quenched through incubation with $3 \%$ hydroxyl peroxide for $10 \mathrm{~min}$ at room temperature. Sections were then incubated with $10 \%$ goat serum (Fuzhou Maixin Biotech Co., Ltd., Fuzhou, China) for $20 \mathrm{~min}$ at room temperature to block nonspecific binding, followed by overnight incubation with the anti-TMEM31 primary antibody (dilution 1:200; \#HPA0527601; Sigma-Aldrich, Merck Millipore, Darmstadt, Germany) at $4^{\circ} \mathrm{C}$. Following incubation with biotinylated goat anti-rabbit IgG (dilution 1:5,000; \#KIT9710; Fuzhou Maixin Biotech Co., Ltd.) secondary antibody for $1 \mathrm{~h}$ at room temperature, the sections were visualized with streptavidin-anti-biotin peroxidase (Fuzhou Maixin Biotech Co., Ltd.). Subsequently, 3,3'-diaminobenzidine was added to the sections for $10 \mathrm{~min}$ or until a color change was observed under a light microscope (Leica Microsystems $\mathrm{GmbH}$, Wetzlar, Germany). Finally, the sections were counterstained with hematoxylin, fixed with a series of ethanol and xylene, and mounted using VectaMount AQ Aqueous Mounting Medium (Vector Laboratories, Inc., Burlingame, CA, USA). In the negative control for immunohistochemical analysis, the primary antibody was replaced with normal IgG (dilution 1:500; cat. no. ab172730; Abcam, Cambridge, UK).

Evaluation of immunostaining. The degree of immunohistochemical staining was evaluated and scored by two pathologists. The proportion of TMEM31-expressing tumor cells was scored as follows: 0, no staining; $1, \leq 30 \% ; 2,31-60 \%$; and 3, 61-100\%. Cells were considered TMEM31-positive if staining was notable in the cytoplasm, regardless of the intensity.

Semiquantitative analysis of the intensity of TMEM31-positive staining in malignant melanoma tissues. The intensity of TMEM31-positive staining in the malignant melanoma tissues was analyzed through integrated optical density (IOD) using Image-Pro ${ }^{\circledR}$ Plus software (version 5.1; Media Cybernetics, Inc., Rockville, MD, USA). All images were taken using the same light microscope and camera sets (Leica Microsystems $\mathrm{GmbH})$. The average IOD/TMEM31-positively stained area $\left(\mu \mathrm{m}^{2}\right)$ was calculated for positively stained tissues.

Statistical analysis. Results are presented as the mean \pm standard error. All statistical analyses were performed using SPSS software (version 17.0; SPSS, Inc., Chicago, IL, USA). A Mann-Whitney U test was performed to compare TMEM31 staining in metastatic and primary melanomas. The Pearson $\chi^{2}$ test was used to determine the association between TMEM31 expression and the clinicopathological characteristics of patients with melanoma. $\mathrm{P}<0.05$ was considered to indicate a statistically significant difference.

\section{Results}

Expression of TMEM31 mRNA in healthy human tissue. TMEM31 mRNA expression was determined via RT-PCR in 
TMEM31

GAPDH

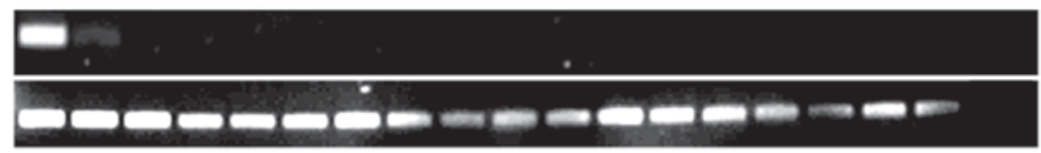

Figure 1. Expression of TMEM31 mRNA in 18 healthy human tissues. Lanes: 1, testis; 2, placenta; 3, lung; 4, spleen; 5, heart; 6, skeletal muscle; 7, prostate; 8, stomach; 9, small intestine; 10, uterus; 11, colon; 12, bone marrow; 13, brain; 14, kidney; 15, liver; 16, thyroid; 17, ovary; 18, bladder; and 19, negative control. TMEM31, transmembrane protein 31 .

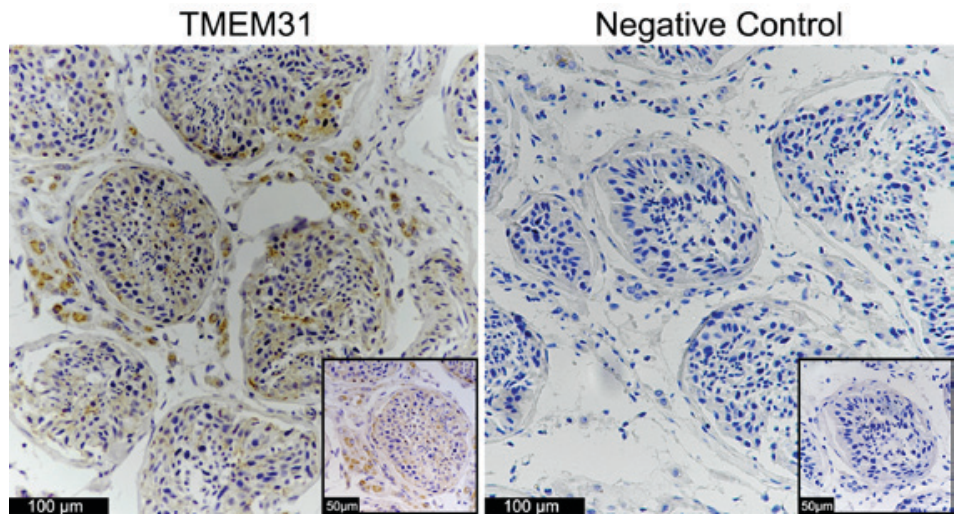

Figure 2. Immunohistochemical analysis of TMEM31 protein expression in healthy human testis. Cytoplasmic staining of TMEM31 was markedly observed in the leydig cells and weakly observed in the inner area of the seminiferous tubules. Objective, x20 (inset, x40). TMEM31, transmembrane protein 31.

the following 18 healthy tissues: Testis, placenta, lung, spleen, heart, skeletal muscle, prostate, stomach, small intestine, uterus, colon, bone marrow, brain, kidney, liver, thyroid, ovary and bladder. TMEM31 was identified to be strongly expressed in testis and weakly expressed in the placenta, but was undetectable in any of the other tissues (Fig. 1). These results correspond with expression data on the UniGene database.

Expression of TMEM31 protein in healthy testis tissue. Immunohistochemical staining for TMEM31 protein was performed using human testis tissue microarray (Fig. 2). TMEM31 protein was markedly expressed in the cytoplasm of leydig cells and weakly expressed in the inner region of seminiferous tubules. By contrast, this staining was not observed when primary antibody was replaced by normal IgG.

Expression of TMEM31 protein in primary melanoma, metastatic melanoma and adjacent healthy tissues. Immunohistochemical staining of melanoma samples was performed (Fig. 3). TMEM31 protein cytoplasmic staining was identified in $120 / 190(63.16 \%)$ melanoma specimens. In total, 55/126 (43.65\%) primary and 15/64 (23.44\%) metastatic melanoma specimens did not stain positive for TMEM31 protein (Fig. 3A and F; Table I). Positively stained primary vs. metastatic melanoma tissues were scored as follows: 1, $27(21.43 \%)$ vs. 15 (23.44\%) (Fig. 3B and G); 2, 20 (15.87\%) vs. 9 (14.06\%) (Fig. 3C and H); and 3, $24(19.05 \%)$ vs. $25(39.06 \%)$ (Fig. 3D and I) (Table I). In addition, no healthy skin tissue was identified to express cytoplasmic TMEM31 protein (Fig. 3E and J). Semiquantitative IOD analysis revealed significantly stronger TMEM31 staining in metastatic melanomas compared with primary melanomas $(0.24 \pm 0.03$ vs. $0.09 \pm 0.01$ IOD/area; P<0.001; Fig. 3K).
Association between TMEM31 protein expression and the clinicopathological characteristics of patients with melanoma. The association between TMEM31 protein expression and the clinicopathological characteristics of patients with melanoma, including gender, age and disease status was investigated. Melanoma samples were divided into groups based on gender (male and female) and age ( $\leq 60$ and $>60$ years old). Immunohistochemical staining revealed that expression of TMEM31 protein was not associated with gender $(\mathrm{P}=0.912)$ or age $(\mathrm{P}=0.615$; Table I). Cytoplasmic staining for TMEM31 protein was present in 71/126 (56.35\%) primary melanomas and 49/64 (76.56\%) metastatic melanomas. A significant difference was identified between the expression of TMEM31 protein in primary and metastatic melanoma specimens $(\mathrm{P}=0.017$; Table I).

\section{Discussion}

Melanoma is responsible for the majority of skin cancer-associated mortalities, and, excluding female lung cancer, is the malignancy with the highest increase in incidence worldwide (1). It is estimated that the median life expectancy of patients with advanced stage melanoma is $<1$ year, as existing therapies are not effective once the tumor has spread to vital organs (15). The metastatic capacity of the tumor is the key prognostic factor for melanoma; however, the molecular mechanisms underlying melanoma metastasis remain poorly understood $(2,16)$. Therefore, the identification and characterization of novel diagnostic and prognostic markers is required, in order to better manage the treatment of patients with melanoma.

CT antigens have been demonstrated to serve as a unique class of tumor-associated antigens, which are expressed 
Table I. Association between TMEM31 protein expression and the clinicopathological characteristics of patients with primary or metastatic melanoma.

TMEM31 IHC staining score [no. of patients (\%)]

\begin{tabular}{|c|c|c|c|c|c|c|}
\hline $\begin{array}{l}\text { Clinicopathological } \\
\text { characteristic }\end{array}$ & $\begin{array}{l}\text { No. of } \\
\text { patients }\end{array}$ & 0 & 1 & 2 & 3 & P-value \\
\hline Gender & & & & & & 0.912 \\
\hline Male & 96 & $35(36.5)$ & $17(17.7)$ & $12(12.5)$ & $32(33.3)$ & \\
\hline Female & 94 & $35(37.2)$ & $25(26.6)$ & $17(18.1)$ & $17(18.1)$ & \\
\hline Age (years old) & & & & & & 0.615 \\
\hline$\leq 60$ & 126 & $48(38.1)$ & $25(19.8)$ & $21(16.7)$ & $32(25.4)$ & \\
\hline$>60$ & 64 & $22(34.3)$ & 17 (26.6) & $8(12.5)$ & 17 (26.6) & \\
\hline Disease status & & & & & & 0.017 \\
\hline Primary & 126 & $55(43.7)$ & $27(21.4)$ & $20(15.9)$ & $24(19.0)$ & \\
\hline Metastasis & 64 & $15(23.4)$ & $15(23.4)$ & $9(14.1)$ & $25(39.1)$ & \\
\hline
\end{tabular}

TMEM31, transmembrane protein 31; IHC, immunohistochemistry.
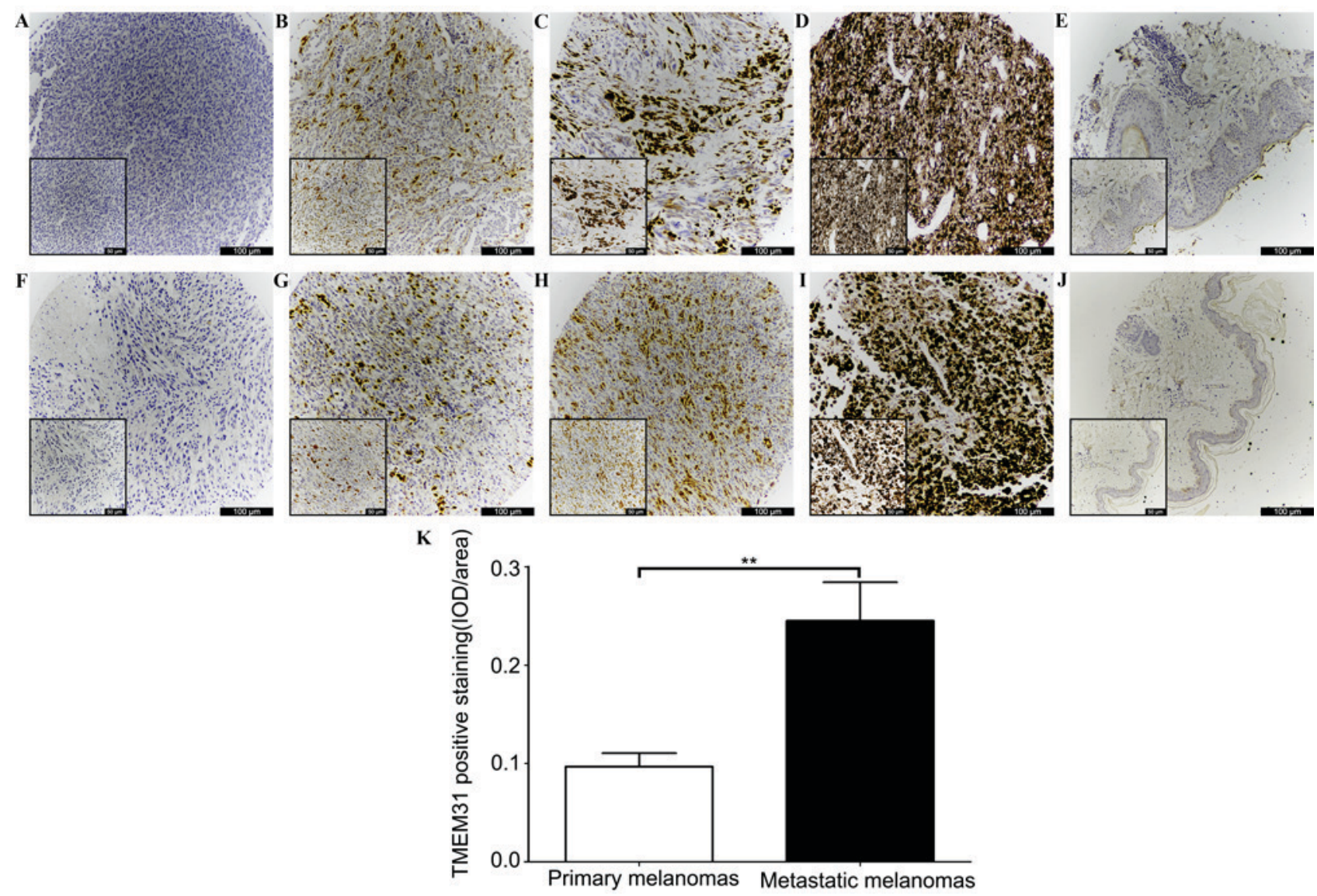

Figure 3. Immunohistochemical analysis of TMEM31 protein expression in primary melanoma, metastatic melanoma and adjacent healthy tissues in a tissue microarray. Primary melanoma tissues with the following staining scores: (A) 0; (B) 1; (C) 2; and (D) 3. (E) There was no TMEM31 staining present in adjacent healthy tissue. Metastatic melanoma tissues with the following staining scores: (F) 0; (G) 1; (H) 2; and (I) 3. (J) There was no TMEM31 staining present in adjacent healthy tissue. Objective, $\mathrm{x} 10$ (inset, $\mathrm{x} 20$ ). (K) Semiquantitative IOD analysis of TMEM31-positive staining in primary and metastatic melanoma samples $\left(0.09 \pm 0.01\right.$ vs. $0.24 \pm 0.03 \mathrm{IOD} /$ area). ${ }^{* *} \mathrm{P}<0.001$. TMEM31, transmembrane protein $31 ; \mathrm{IOD}$, integrated optical density.

in different histological types of tumor, but not in healthy tissues, excluding testis and placenta tissue $(5,6)$. Since testes are immune privileged and do not express human leukocyte antigen molecules, CT antigens are considered to be clinically important biomarkers of cancer in this organ and immunotherapeutic targets $(6,7)$. It has been suggested that $\mathrm{CT}$ antigen 
genes that are located on the $\mathrm{X}$ chromosome frequently form multigene families and exhibit tissue-restricted expression (4). A previous study identified 153 CT antigens, 83 of which were located on the X chromosome (5). A preliminary study identified a testis-specific gene, TMEM31, in addition to 291 human testis-specific genes that were all encoded by the genes on the $\mathrm{X}$ chromosome (17). In the present study, it was demonstrated that in healthy tissue TMEM31 is predominantly expressed in testes and weakly expressed in the placenta. In addition, it was revealed that TMEM31 exhibits significantly increased expression in metastatic melanoma compared with primary melanoma. These results suggest that TMEM31 is a novel CT antigen.

To the best of our knowledge, the present study reported for the first time the expression of TMEM31 protein in melanoma. TMEM31 protein expression in melanoma samples in the present study is notable compared with other known potential CT antigens. In a previous study, the well-characterized CT antigen NY-ESO-1 was demonstrated to be expressed in $30 \%$ of stage I and $40 \%$ of stage II melanomas tested (18). Other studies revealed that NY-ESO-1 was detected in 36 (11), 31.6 (12) and 45\% (14) of melanoma samples tested. In addition, MAGE-C1/CT7 and MAGE-C2/CT10 protein expression has been identified in 24 and $33 \%$ of primary melanoma tissues, respectively (19), and MAGE-A1 protein expression has been detected in $27 \%$ of metastatic melanoma tissues (13). Furthermore, paraffin-embedded melanoma tissue samples were analyzed using immunohistochemistry for the presence of MAGE-A1 and MAGE-A4 protein. The frequency of the presence of MAGE-A1 and MAGE-A4 proteins in 586 melanoma samples was identified to be 37 and $29 \%$, respectively (14). In contrast, the present study identified TMEM31 protein expression in 120/190 (63.16\%) melanoma tissues, irrespective of histotype, stage and clinical grade, which indicates that it is a diagnostic biomarker and therapeutic candidate for melanoma.

TMEM31 protein expression was significantly higher in metastatic compared with primary melanoma. A previous study revealed a clear correlation between $\mathrm{CT}$ antigen expression and advanced tumor stage. The frequency of CT antigen expression in primary tumors for MAGE-A1 and MAGE-A4 was lower (20 and 9\%, respectively). MAGE-A1 and MAGE-A4 expression levels were increased with advancing disease stage (to 51 and $44 \%$ in distant metastases, respectively) (14). In the present study, TMEM31 protein expression significantly increased from $56.35 \%$ in primary melanoma samples to $76.56 \%$ in metastatic melanoma samples $(\mathrm{P}=0.017)$. The results of the present study suggest that TMEM31 is a novel CT antigen gene, with particularly high expression in melanoma. In addition, TMEM31 protein expression was significantly increased in metastatic compared with primary melanoma, suggesting that it is potential therapeutic target for the inhibition of tumor metastasis pathways. In conclusion, TMEM31 may have applications in the diagnosis, prognosis and immunotherapeutic treatment of patients with melanoma.

\section{References}

1. Jemal A, Siegel R, Xu J and Ward E: Cancer statistics, 2010. CA Cancer J Clin 60: 277-300, 2010.

2. Fernandez-Flores A: Prognostic factors for melanoma progression and metastasis: From Hematoxylin-Eosin to genetics. Rom J Morphol Embryol 53: 449-459, 2012.

3. Ives NJ, Stowe RL, Lorigan P and Wheatley K: Chemotherapy compared with biochemotherapy for the treatment of metastatic melanoma: A meta-analysis of 18 trials involving 2,621 patients. J Clinc Oncol 25: 5426-5434, 2007.

4. Simpson AJ, Caballero OL, Jungbluth A, Chen YT and Old LJ: Cancer/testis antigens, gametogenesis and cancer. Nat Rev Cancer 5: 615-625, 2005.

5. Song MH, Choi KU, Shin DH, Lee CH and Lee SY: Identification of the cancer/testis antigens AKAP3 and CTp11 by SEREX in hepatocellular carcinoma. Oncol Rep 28: 1792-1798, 2012.

6. Scanlan MJ, Simpson AJ and Old LJ: The cancer/testis genes: Review, standardization, and commentary. Cancer Immun 4: 1, 2004.

7. Brichard VG and Lejeune D: Cancer immunotherapy targeting tumour-specific antigens: Towards a new therapy for minimal residual disease. Expert Opin Biol Ther 8: 951-968, 2008.

8. Van der Bruggen P, Traversari C, Chomez P, Lurguin C, De Plaen E, Van den Eynde B, Knuth A and Boon T: A gene encoding an antigen recognized by cytolytic $\mathrm{T}$ lymphocytes on a human melanoma. Science 254: 1643-1647, 1991.

9. Grigoriadisa A, Caballerob OL, Hoekc KS, da Silva L, Chen YT, Shin SJ, Jungbluth AA, Miller LD, Clouston D, Cebon J, et al: CT-X antigen expression in human breast cancer. Proc Natl Acad Sci USA 106: 13493-13498, 2009.

10. Hofmann O, Caballero OL, Stevenson BJ, Chen YT, Cohen T, Chua R, Maher CA, Panji S, Schaefer U, Kruger A, et al: Genome-wide analysis of cancer/testis gene expression. Proc Natl Acad Sci USA 105: 20422-20427, 2008.

11. Jungbluth AA, Chen YT, Stockert E, Busam KJ, Kolb D, Iversen K, Coplan K, Williamson B, Altorki N and Old LJ: Immunohistochemical analysis of NY-ESO-1 antigen expression in normal and malignant human tissues. Int J Cancer 192: 856-860, 2001

12. Bolli M, Schultz-Thater E, Zajac P, Guller U, Feder C, Sanguedolce F, Carafa V, Terracciano L, Hudolin T, Spagnoli GC and Tornillo L: NY-ESO-1/LAGE-1 coexpression with MAGE-A cancer/testis antigens: A tissue microarray study. Int J Cancer 115: 960-966, 2005.

13. Gajjar NA, Cochran AJ and Binder SW: Is MAGE-1 expression in metastatic malignant melanomas really helpful? Am J Surg Pathol 28: 883-888, 2004.

14. Barrow C, Browning J, MacGregor D, Davis ID, Sturrock S, Jungbluth AA and Cebon J: Tumor antigen expression in melanoma varies according to antigen and stage. Clin Cancer Res 12: 764-771, 2006.

15. Berwick $M$ and Wiggins $C$ : The current epidemiology of cutaneous malignant melanoma. Front Biosci 11: 1244-1254, 2006.

16. Palmieri G, Capone M, Ascierto ML, Gentilcore G, Stroncek DF, Stroncek DF, Casula M, Sini MC, Palla M, Mozzillo N and Ascierto PA: Main roads to melanoma. J Transl Med 14: 86, 2009

17. Tang A, Yu Z, Gui Y, Zhu H, Zhang L, Zhang J and Cai Z: Characteristics of 292 testis-Specific genes in human. Biol Pharm Bull 30: 865-872, 2007.

18. Svobodová S, Browning J, MacGregor D, Pollara G, Scolyer RA, Murali R, Thompson JF, Deb S, Azad A, Davis ID and Cebon JS: Cancer-testis antigen expression in primary cutaneous melanoma has independent prognostic value comparable to that of Breslow thickness, ulceration and mitotic rate. Eur J Cancer 47: 460-469, 2011.

19. Curioni-Fontecedro A, Nuber N, Mihic-Probst D, Seifert B, Soldini D, Dummer R, Knuth A, van den Broek M and Moch H: Expression of MAGE-C1/CT7 and MAGE-C2/CT10 predicts lymph node metastasis in melanoma patients. PLoS One 6: e21418, 2011. 